\title{
Virtual 3D Object Imaging for Cultural Artefacts: Demonstrator
}

\author{
R. Collmann \\ Art History Program / eScholarship Research Centre \\ University of Melbourne, Australia \\ r.collmann@unimelb.edu.au
}

\author{
D. van der Knijff \\ ITS Research Services \\ University of Melbourne, Australia \\ dirk@unimelb.edu.au
}

$3 D$ visualisation. 3D photography. Stereoscopic photography. Archaeology.

\begin{abstract}
Following the manufacture of the Virtual 3D Object Rig Model 1 (V3DORm1) in 2008 (http://www.versi.edu.au/3d) and subsequent testing in the Australian Institute of Archaeology (AIA) in Melbourne, an advanced Virtual 3D Object Rig Model 2 (V3DORm2) has been designed and manufactured in the La Trobe University Department of Physical Sciences Engineering Laboratory and is being currently tested.

The V3DORm2 takes multiple individual 2D images from various camera perspective angles of archaeological artefacts for use in constructing QTVR-type 'virtual' 3D .MOV files which manifest 3D appearance via visual monocular depthperception cues when the photographed object is moved by user control. In addition to the highdefinition digital single-shot cameras already employed, the new apparatus can use a HD3D video camera to add further realism to the $3 D$ moving images which can be produced using a variety of image file techniques.
\end{abstract}

A server-side image delivery mechanism for selective image download (similar to Google Earth) will be demonstrated on-line. This has utility for low-bandwidth users and for collections wishing to protect institutional IP.

A video outlining full V3DOR workflow processes will be shown as well as the outcomes of artefact imaging on an auto-stereo monitor.

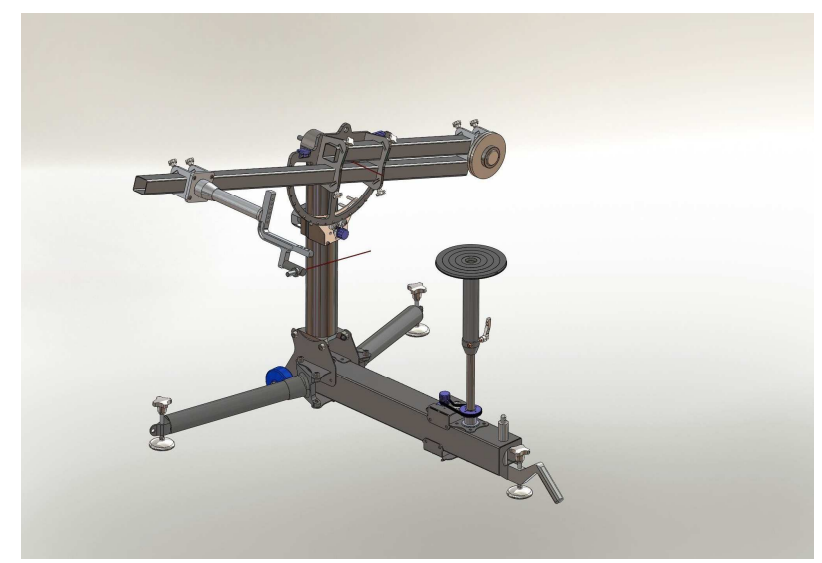

Figure 1: V3DORm2 CAD 